\section{Evolution: students debate the debate}

I asked my third- and fourthyear undergraduate students whether they thought that evolutionary theory needs rethinking (see Nature 514, 161$164 ; 2014)$. More than two-thirds (26 out of 38 ) argued that it did not - because the synthesis proposed by Kevin Laland et al. has largely already occurred.

Far from being neglected as Laland and colleagues imply, topics such as developmental bias, plasticity, niche construction and extra-genetic inheritance are well established in basic courses on evolutionary theory. Students today recognize that these processes can be both outcomes and causes of evolution. There is also a large body of work on co-evolutionary dynamics and interacting phenotypes (see, for example, any of the 400 or so papers that cite J. B. Wolf et al. Trends Ecol. Evol. 13, 64-69; 1998).

Although all of my students agreed that the phenomena discussed by Laland and colleagues warrant further study, they - like the authors of the counterpoint piece, Gregory Wray et al. - did not view the authors' ideas as an "alternative vision of evolution". There would therefore seem to be no "struggle for the very soul of the discipline".

Hope Klug University of Tennessee, Chattanooga, USA. hope-klug@utc.edu

\section{Evolution: viruses are key players}

The debate on rethinking evolutionary theory (see Nature 514, 161-164; 2014) should include viruses. By integrating into host DNA, viruses have markedly influenced the evolution and development of cellular organisms (see, for example, F. Baluška Ann. NY Acad. Sci. 1178, 106-119; 2009).

Viruses are the most abundant genetic entities on the planet. Almost all genomes of cellular organisms contain viral sequences, elements of which are now essential in gene regulation.

Persistent endogenous retroviruses, for example, have contributed crucially to the evolution of the mammalian placenta. And the genetic variations that led to the evolution of adaptive immunity in vertebrates, or the equivalent system in prokaryotes, were not a result of random errors in DNA replication but of viral infection events (see L. P. Villarreal Viruses 3, 1933-1958; 2011).

Guenther Witzany Telos-

Philosophische Praxis, Bürmoos, Austria.

František Baluška University of Bonn, Germany.

baluska@uni-bonn.de

\section{Evolution: networks and energy count}

Standard evolutionary theory should incorporate the complexity of adaptive evolving systems - including species, niches and environment - as dynamic relationship networks (see Nature 514, 161-164; 2014).

For example, epigenetic inheritance - which changes gene expression but not the DNA sequence - involves the storage of molecular information and its retrieval, transfer and processing at the supramolecular level. This involves transitory processes that are self-organized, selfassembled and dynamic.

DNA replication too is one of countless functional tasks of interest in the study of evolution: changes propagate through interlinked levels of organization, inducing connectivity and interaction at all scales of the multilevel system.

The process of natural

\section{- NATURE.COM}

For more on the evolutionary theory debate, see: go nature.com/gbqfv9 selection is now being captured, by modelling fitness attractors that incorporate power laws and non-equilibrium steady states at the edge of chaos, with energy landscapes made of basins, valleys, floors, ridges and saddle points (see, for example, K. Friston J. R. Soc. Interface 10, 20130475; 2013).

Arturo Tozzi ASL Napoli 2 Nord, Naples, Italy.

tozziarturo@libero.it

\section{Anti-vivisectionists respond}

Following our seven-month undercover investigation, the British Union for the Abolition of Vivisection (BUAV) strongly disagrees with your claim that the Max Planck Institute in Tübingen, Germany, has done a "good job" on its website in explaining its neuroscience research on macaques (see Nature 513, 459-460; 2014).

Our investigation of the macaques' treatment and conditions was undertaken with SOKO-TS, a German animalprotection organization. The BUAV goes to enormous lengths to check facts and is extremely careful only to publish allegations that it believes are demonstrably true.

After rigorously scrutinizing footage and documentation from this investigation, the leading German television station Stern has called into serious question claims and images posted on the Max Planck Institute's website. For example, the institute makes what in our opinion is the bizarre claim on its website that the animals do not suffer.

Jane Goodall, the renowned primatologist, says she has seldom seen such sickening experiments. They have no place in a civilised society.

Following the Stern broadcast, the institute has identified a need for improvement in terms of staff organization and agreed to introduce overnight care for the animals following surgery and to improve veterinary attention.

We consider that the use of macaques in these experiments is unnecessary: the continued creative and ethical use of imaging techniques on patients and volunteers is, we believe, far more likely to produce improvements in neurological health.

It is not better PR that animal researchers need, as you argue, but a paradigm shift in thinking, a better appreciation of the suffering they cause animals and a commitment to genuine transparency.

Michelle Thew BUAV, London, UK.

michelle.thew@buav.org

\section{Ice-bucket challenge should jolt funding}

The Italian prime minister Matteo Renzi was among the vast number of people who accepted the ice-bucket challenge' this summer, helping to raise $€ 2$ million (US\$2.5 million) in Italy for research into amyotrophic lateral sclerosis (ALS), also known as motor neuron disease (see Nature 514, 403-404; 2014).

This sum exceeds his government's average annual budget for ALS research, which is still seriously underfunded - despite Italy ranking third in international ALS publications, after the United States and Japan.

ALS researchers worldwide are waiting to see how the sum of around $\$ 100$ million that has been collected by this philanthropic phenomenon will be used, and whether it will boost governments' plummeting contributions towards basic research. I hope so: without such funds there can be no development of new drugs for this incurable disease.

Maria Teresa Carrì University of Rome 'Tor Vergata', Italy.

carri@bio.uniroma2.it 